\title{
SIMULATORS AND SIMULATIONS: THEIR CHARACTERISTICS AND APPLICATIONS TO THE SIMULATION AND CONTROL OF AEROSPACE VEHICLES
}

Copyright (C) 2003 Society of Automotive Engineers, Inc

\begin{abstract}
In this work we discuss some types of simulators and simulations, their characteristics and applications to the simulation and control of aerospace vehicles. This includes: the basic definitions, types and characteristics of simulators and simulations (physical, computational, hybrid, etc.; discrete events, discrete time, continuous time, etc; deterministic, stochastic, etc.) their basic compromise (simplicity $\mathrm{x}$ fidelity), their man-machine interfaces and interactions (virtual, constructive, live, etc.), their evolution law (time, events, mixed, etc.), their architectures ("stand-alone", PIL, HIL, MIL, DIS, HLA, etc.), their environments (discrete, continuous, hybrid, etc.) and their applications to the simulation and control of aerospace vehicles. This is illustrated by some examples driven from the aerospace industry

\section{INTRODUCTION}

Since its beginning, the aerospace industry became one of the main users and beneficiaries of simulation, stimulating and permitting the development of tools even more powerful. Successively, it took advantage of the use of: physical simulation for design and training; simulation in analog computers for control systems; and, in the age of digital computers, digital simulation in the remaining areas of knowledge involved in the diverse phases of development of aerospace vehicles. Today, simulation is recognized even more as an essential tool for the diverse phases of product development such as: specification, design, development, production, tests, accreditation, maintenance, training, and marketing.
\end{abstract}

\author{
Marcelo Lopes de Oliveira e Souza \\ National Institute for Space Research - INPE \\ Gilberto da Cunha Trivelato \\ Empresa Brasileira de Aeronáutica S. A. - EMBRAER
}


IDENTIFICATION: Is the act of doing a description of (relations of) a system, process, phenomenon, being or entity in a midia, with a language, and with an objective or viewpoint, based in measures of its variables and even some of its parameters. A special but very common form of identification is:

DATA REDUCTION: Is the act of doing the concen tration of the information contained in a large set of data into a small set of data that characterizes or, at least, takes part in the characterization of a model of a system, process, phenomenon, being or entity.

IDENTITY/IDENTIFIED MODEL: Is the descripti on done by identification. It can be: physical, mathemati cal, logical, computational.

IDENTIFIER: Is the agent that does the identifica tion.

TYPES, CHARACTERISTICS, METHODS OF IDENTIFICATIONS/IDENTIFIED MODELS: The most common types of mathematical identifications/identified models are:

- Parametric and non parametric;

- In the domain of time or frequency;

- Sequential or in batch;

- Deterministic or stochastic;

BASIC COMPROMISE: The basic compromise of any identification is: simplicity $\mathrm{X}$ fidelity.

\section{MODELS OF VEHICLES: REQUIREMENTS OF THE AEROESPACE INDUSTRY}

To be used in all phases of development of an aerospa ce vehicle, the models simulated must include all pertinent systems and must allow the adjustment of the complexity of each subsystem according to the application desired. To meet these requirements, in a general form, the simulation of an aircraft must contain: the models of the atmosphere, engines, aerodynamics, motion, other subsystems, landing gear, controls, graphical user interfaces-GUI(pilot displays, monitoring interfaces or data communication interfaces), and initialization routines. A common generic model of an aircraft is shown in Figure 1.

It should also be possible to include and to exclude models of specific parts whenever necessary (e.g. of the electric system). a fundamental property of these simulated systems is to allow us to change blocks of the same subsystem, but with different levels of fidelity. a practical example on the need of different levels of simulation of the same subsystem can be verified in the use of the simulated electrical system when we are analysing the control system of an aircraft or of a satellite: in the first case, the batteries can be modeled and simulated by state machines with on and off levels; while in the second case, the battery charge level must be included in the model because it changes the capacity of the actuators (ex.:magnetic coils)then changing the control parameters. the investigation on the effects of the sampling period of aerospace vehicle control systems on the vibration modes of flexible structures had its impor tance increased recently due to the use of larger and lighter structures in satellites and due to the design of lighter and thinner aircraft wings.

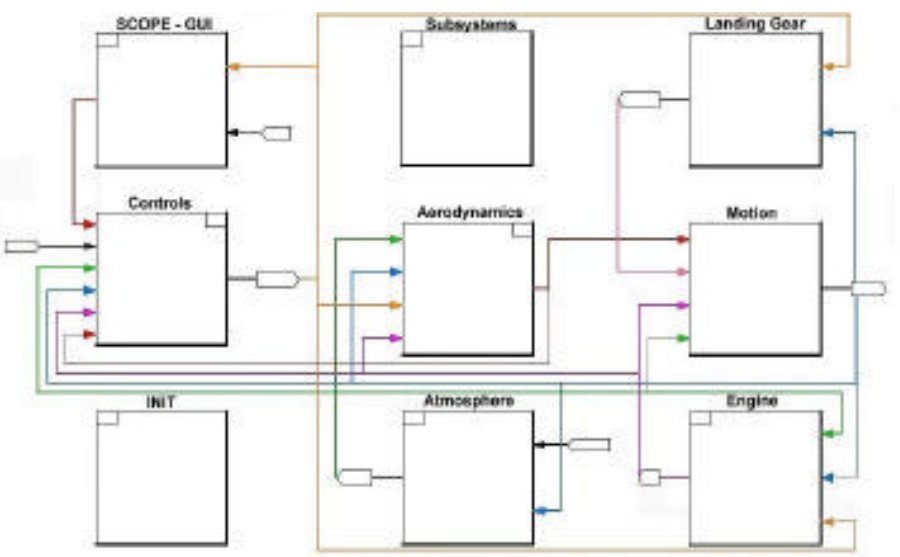

Figure 1: General model of an aircraft for computational simulators.

\section{SIMULATIONS AND SIMULATORS: BASIC CON CEPTS,TYPES,CHARACTERISTICS, ETC.}

SIMULATION: Is the act of doing the description of the evolution of a system, process, phenomenon, being or entity in a midia, with a language, and with an objective or viewpoint.

SIMULATOR: Is the agent that does the simulation.

BASIC COMPROMISE: the basic compromise of any simulation is: simplicity $\mathrm{x}$ fidelity.

TYPES, CHARACTERISTICS, METHODS OF SIMULATIONS/SIMULATORS: The most common types of mathematical simulations/simulators classified by/are:

- Models: physical, mathematical,logical,computational;

- Evolutive/evolving formalism: continuous time $t$, discrete time $\mathrm{t}_{\mathrm{k}}=\mathrm{k} . \mathrm{t}$, discrete events $\mathrm{e}_{\mathrm{k}}: \mathrm{x}(\mathrm{k} . \mathrm{t})=\mathrm{x}_{\mathrm{k}}$;

- Relation simulated time $\tau$ / real time t: virtual time $\tau \neq$ $\mathrm{t}$, real time $\tau=\mathrm{t}$, more than real time $\tau>\mathrm{t}$ ("fast motion, fight time"), less than real time $\tau<\mathrm{t}$ ("slow motion");

- Interactions H-M: construtive V-V, virtual R-V, live R-R;

- Interfaces/languages: physical $\mathrm{x}$ virtual/commands $\mathrm{x}$ visual. 


\section{SIMULATIONS AND SIMULATORS: REQUIRE MENTS OF THE AEROSPACE INDUSTRY}

The occurrence of use of new materials, of new appli cations, of reduction in weight and consumption, of search for optimizing such vehicle designs, and the need of reduc ing the production cycle(see Figs.2,3)created new problems with distinct characteristics (see Figs.4-7, Tables 1,2), that did not exist or that were neglected before, and that can be
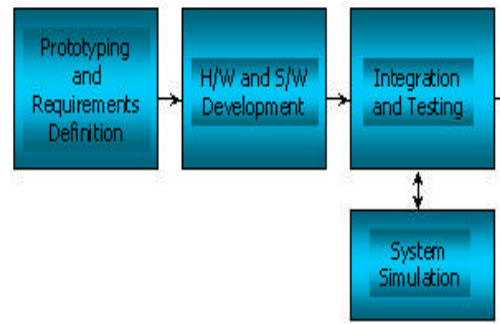

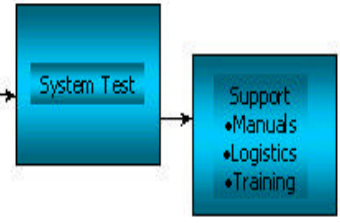

Figure 2: Production cycle/development phases of an aeroespace vehicle supported by simulations.

\section{SIM ovolution during the design process}

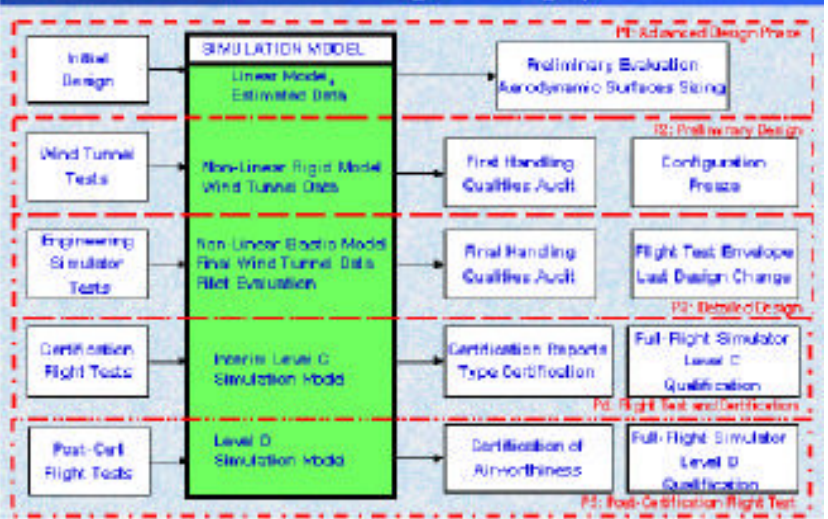

Figure 3: Simulations used in the production cycle/develop ment phases of an aeroespace vehicle.

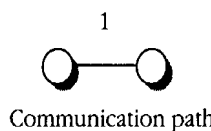

with two programmers
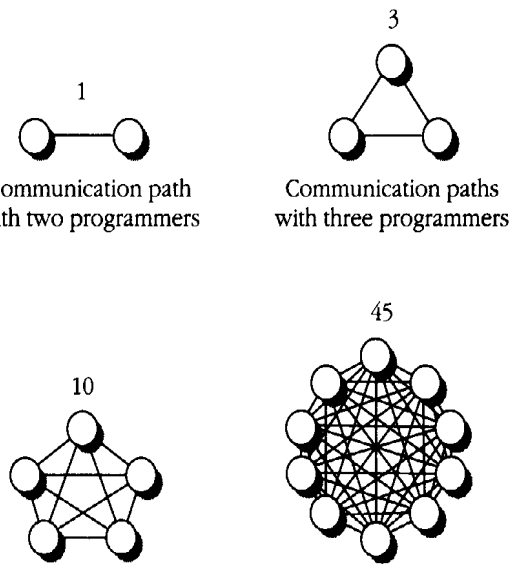

Communication paths with five programmers
45

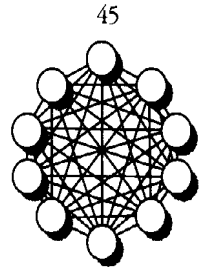

Communication paths with ten programmers
Figure 4: Characteristics: combinatorial growth of mutual communication of programmers may increase unreliability. solved with the support of simulation and rapid prototyp ing, including those simulations with hardware or even pi lots in the loop. These characteristics (see Figs. 4-7, Tables 1,2 ) made it fundamental the reuse of models of the diverse systems along the diverse phases of the product (see Figs.2, 3 ) as a way of reducing time and costs, increasing the relia bility, the ease of accreditation, the fidelity for training, the reproduction and investigation of faults or accidents, etc.

\section{Effort Distribution}

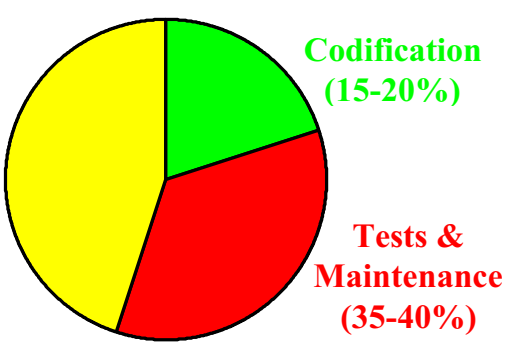

Figure 5: Characteristics: Percentage of effort distribution in commercial systems.

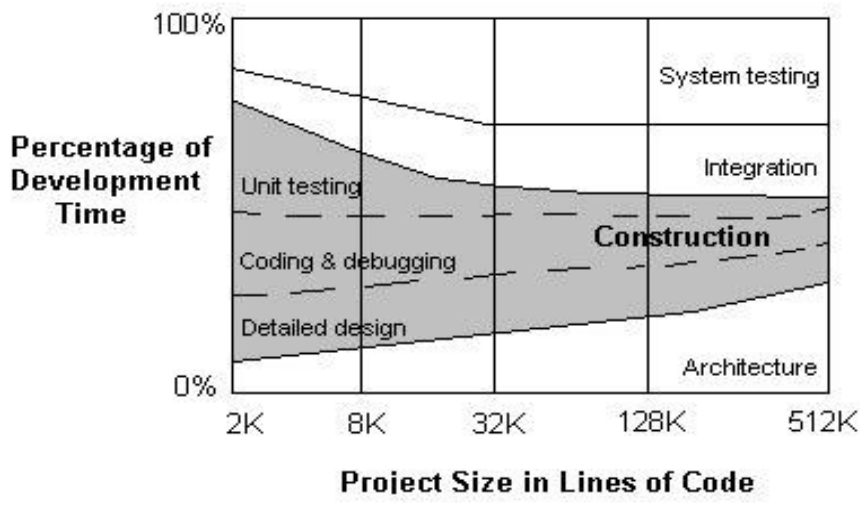

Figure 6: Characteristics: percentage of development time versus the number of lines of code in commercial systems.

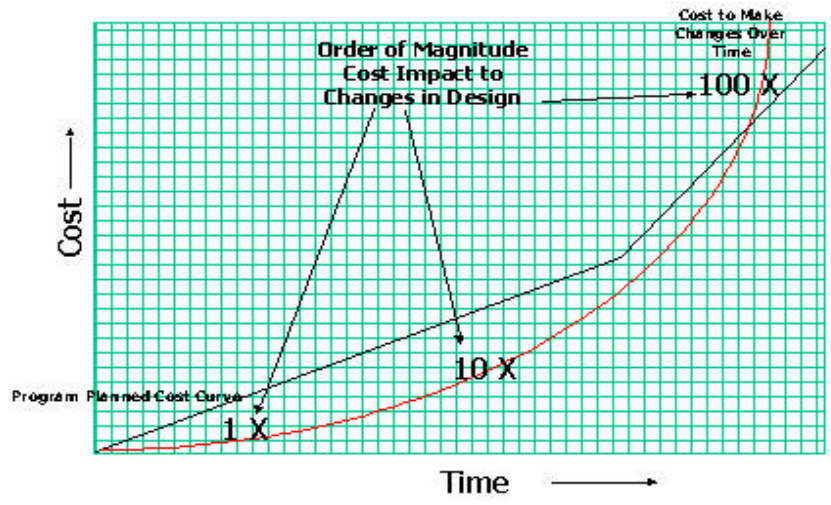

Figure 7: Characteristics: higher cost changes versus time in commercial systems. 
Table 1: Characteristics: exponential growth of number of lines of code with model/time

\begin{tabular}{|l|l|l|l|l|l|}
\hline Model & $\begin{array}{l}\text { F-15 } \\
\text { EAGLE } \\
\text { (ACS) }\end{array}$ & $\begin{array}{l}\text { ALX } \\
\text { (basic) }\end{array}$ & F-22 & JSF & Next \\
\hline Code Lines & 12.000 & 250.000 & 1.500 .000 & 3.500 .000 & $? ? ?$ \\
\hline
\end{tabular}

Table 2:Characteristics:High reliab./cost of accidents/faults

\begin{tabular}{|l|l|l|l|l|}
\hline $\begin{array}{l}\text { Accidental } \\
\text { Fault }\end{array}$ & $\begin{array}{l}\text { Denver } \\
\text { Airport }\end{array}$ & Ariane 5.01 & Chernobyl: & Next \\
\hline Cost & US\$300.000. & $\begin{array}{l}\text { US\$2.000.000 } \\
.000\end{array}$ & 100.000 lives & $? ? ?$ \\
\hline
\end{tabular}

\section{MODELING, IDENTIFICATION AND SIMULATION ENVIRONMENTS: BASIC CONCEPTS, TYPES, CHARACTERISTICS, ETC.}

MIS ENVIRONMENT: Is an integrated set of physi cal, mathematical, logical, computational, etc. resources, to model or identify a system, process, phenomenon, being or entity and/or simulate its evolution in a midia, with a lan guage, and with an objective or viewpoint.

BASIC COMPROMISE: the basic compromise of any MIS environment is: simplicity $\mathrm{x}$ fidelity.

TYPES AND CHARACTERISTICS OF MIS ENVI RONMENTS: The most common types of MIS environments classified by/are:

- Models: physical, mathematical,logical,computational;

- Evolutive/evolving formalism: continuous time t, discrete time $t_{k}=k . t$ (synchronous), discrete events $e_{k}$ : $\mathrm{x}(\mathrm{k} . \mathrm{t})=\mathrm{x}_{\mathrm{k}}$ (asynchronous);

- Relation simulated time $\tau$ / natural time t: virtual time $\tau \neq \mathrm{t}$, real time $\tau=\mathrm{t}$, more than real time $\tau>\mathrm{t}$ (fast mo tion, fight time),less than real time $\tau>\mathrm{t}$ (slow motion);

- Interactions Human-Machine H-M: constructive Virtual-Virtual, Virtual Real-Virtual; live Real-Real;

- Interfaces/lang: physical x virtual/ commands x visual;

- Spatial \& temporal distribution: concentrated ("standalone, computing unit") x parallel, distributed ("com puting cluster, topology,commun..network /protocols";

- Processor/hardware/ men in the loop: 1 processor ("stand-alone") x 2,.., p processors $\mathrm{x} \mathrm{s}$ sensors $\mathrm{x}$ a actuators $\mathrm{x} m$ pilots in the loop;

- Num.of objects/players and interactions: small x mega;

- Specialization of hardwares/softwares/operational sys tems: Computer Off The Shelf-COTS (PCs) x custom;

- Standars/norms/paradigms: physical x languages x pro gramming/documentation/tests $\mathrm{x}$ applications $\mathrm{x}$ envi ronments $\mathrm{x}$ architectures.

CONSIDERATIONS ON TYPES AND CHARACTE RISTICS OF MIS ENVIRONMENTS: The most common considerations on the types and characteristics of MIS environments are:

- Real-time simulation and support for HIL modeling are increasingly recognized as essential tools for aeronautic design. HIL is no longer a luxury in modern system design;

- Simulation software is notoriously difficult to write, debug and maintain;

- Real-time, high-performance, deadlock-free distri buted code is even harder;

- Softwares automatically generates, downloads and runs code that meets above-mentioned constraints, without forcing the user to write any target code.;

- COTS software and hardware allow large savings in cost and engineering effort cots software has replaced custom development;

- PCs have now supplanted workstations and even main frames for many simulation and control applications;

- PC clusters, embedded PCs, high-end graphics cards and other technologies increased the range of applications that can benefit from the low cost of COTS hardware;

- Commercial simulation packages such as Matlab/Simulink ${ }^{\mathrm{TM}}$ and Matrixx/Systembuild ${ }^{\mathrm{TM}}$ are now widely used in the aeronautics industry. they have become the modeling tools of choice;

- Graphic interface mimics the function-block methodologies taught in universities and technical schools; this reduces the learning time and increases engineering productivity;

- Automatic code generation and documentation; this frees engineers from the tedious and error-prone task of writing code. they make possible to go from concept to simulation without ever having to write code.

\section{MIS ENVIRONMENTS:METHODS \& TECHNIQUES}

- Full nonlinear x linearization around given operatio nal conditions;

- Analytic x numerical solution of algebraic equations and algebraic loops;

- analytic x analog x numerical integration of ordinary differential equations;

- finite elements $\mathrm{x}$ finite differences integration of partial differential equations;

- statistically known x Monte Carlo simulations via design of experiments;

- Data interpolation $\mathrm{x}$ extrapolation via least squares, curve fitting, splines;

- Continuous $\mathrm{x}$ discrete time via discretization in time with sampler \& hold;

- Time driven (synchronous) $\mathrm{x}$ event driven (asynchro nous) $\mathrm{x}$ mix of both;

- Real time $\mathrm{x}$ virtual time(slow xfast motion)simulation; 
- Small simulation $\mathrm{x}$ mega simulation via computing unit $\mathrm{x}$ computing cluster;

- Concentrated $\mathrm{x}$ distributed simulation via $1 \times 2, . ., \mathrm{p}$ processors in the loop;

- Software $\mathrm{x}$ hardware (processors, sensors, actuators) $\mathrm{x}$ men in the loop simulation;

- Sequential $\mathrm{x}$ simultaneous simulation via $1 \times 2, \ldots, \mathrm{p}$ processors+clocks and communic. network/ protocols;

\section{MIS ENVIRONMENTS: REQUIREMENTS OF THE AEROESPACE INDUSTRY}

For the adequate use of simulation environments in the production cycles of an aerospace vehicle it is necessa ry to observe the important aspects of each phase with res pect to those environments. The current designs in this a rea may be developed by teams distributed even in diverse places around Earth, which requires standardization and $\mathrm{u}$ niversality as a necessary requirement for the specification of systems, to have the same meaning among the diverse teams. In some areas, depending on the quality of the mo dels used, the design phase merges with the specification phase. The interoperability of models and of the generated codes is essential for prototyping the systems under diverse technical aspects and in diverse places during the develop ment phase, and to allow adequate environments for the integration and tests of subsystems. These same models must be reused in production and tests devices in the pro duction line.

All these prototypes, in the whole or in part, can and must be used for the accreditation of the product under the correspondent institutions (FAA, JAA, CTA, etc.), for trai ning the members involved during the program,and for training the customers in the operation and maintenance of the final product. It must be possible the reuse and incorpo ration of legacy codes that were already verified, validated and accredited. With the inclusion of these requirements in the beginning of the specification phase, we have in the end truly simulated systems available for the investigation of failures and for maintenance of the product developed. The simulation software can be used for marketing or for support to the sales during product development and in the after-sale. The use of environments that meet these require ments from product conception to after-sale represents significant savings with benefits in the product reliability and in the reduction in the development time(see Figs 8,9).

EXAMPLES OF MIS ENVIR.FOR GENERAL COMP. SIMUL.: MATLAB, MATRIXx, CONSTELLATION

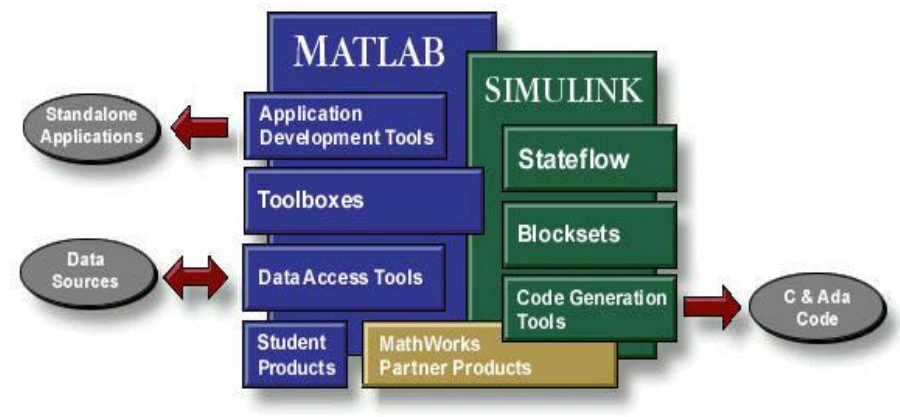

Figure 8: The MATLAB ${ }^{\mathrm{TM}}$ \& family MIS environment.

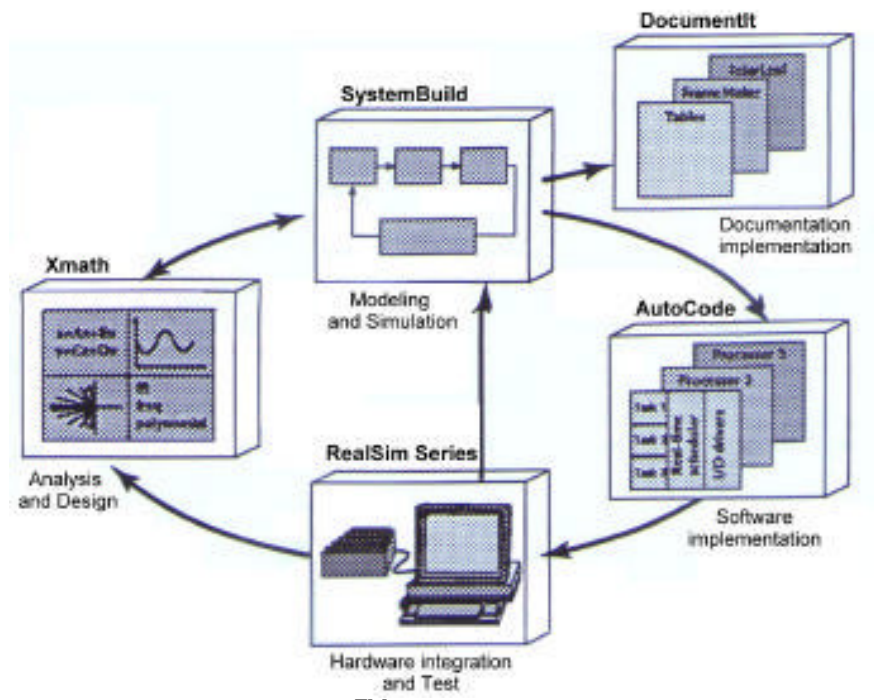

Figure 9: The MATRIXx ${ }^{\mathrm{TM}} \&$ family MIS environment.

Table 1: Comparison of functions of the MATRIXx and MATLAB MIS environments.

\begin{tabular}{|l|l|l|}
\hline Item & MATRIXx & MATLAB \\
\hline Installation & Needs support & Easy \\
\hline Use & Easy and intuitive & Easy and intuitive \\
\hline Platform & DOS/WINDOWS, OpenVMS, UNIX, VxWorks, PSOS & DOS/WINDOWS, OpenVMS, UNIX, VxWorks, Maços (5.3) \\
\hline Support & Very good & Very good \\
\hline Interfaces & Visual, graphical and intuitive & Visual, graphical and intuitive \\
\hline Resources for modeling and simulation & Very good & Very good ;and vast, considering the toolboxes \\
\hline Simulation control & Pause, resume e re-run & Pause, resume e re-run \\
\hline Presentation of results & Graphical objects & Graphical objects \\
\hline Project documentation & Very good & Very good \\
\hline Code documentation & Very good & Poor \\
\hline Code generation & Ce ADA & C (restriction) e ADA \\
\hline Conversion continuous discrete & Automatically (back, forward e Tustin) & Needs inclus. of "sampler e holder " in the model \\
\hline Support to real-time & Yes & Toolbox of third parties \\
\hline
\end{tabular}

